\title{
THE ASYMPTOTIC BEHAVIOR OF A DECOMPOSITION DUE TO HARZHEIM ${ }^{1}$
}

\section{T. S. PITCHER}

We will write $((x))$ for the fractional part of a real number $x$ and $[x]$ for the greatest integer less than or equal to $x$ so that $((x))=x$ $-[x]$. E. Harzheim has shown [2] the existence for each $x$ in $[0,1)$ of a sequence of nonnegative integers $\alpha_{2}(x), \cdots, \alpha_{n}(x), \cdots$ with

$$
\left(\left(x 2^{\alpha_{2}(x)} 3^{\alpha_{3}(x)} \cdots n^{\alpha_{n}(x)}\right)\right)<1 / n .
$$

The $\alpha_{n}$ are determined inductively by setting $\alpha_{n}(x)$ equal to the unique integer $k$ such that

$$
\sum_{j=1}^{k} \frac{1}{n^{j}} \leqq\left(\left(x 2^{\alpha_{2}(x)} \cdots(n-1)^{\alpha_{n-1}(x)}\right)\right)<\sum_{j=1}^{k+1} \frac{1}{n^{j}}
$$

(taking $\left.\sum_{j=1}^{0}=0\right)$. This is always possible since $\sum_{j=1}^{\infty} 1 / n^{j}=1 /(n-1)$. Conversely, given any sequence $\alpha_{2}, \alpha_{3}, \cdots$ of nonnegative integers there is an $x$ in $[0,1)$ with $\alpha_{i}(x)=\alpha_{i}, i=2,3, \cdots$. We are concerned with the probabilistic behavior of the random variables $\alpha_{n}$ and $x_{n}$, $x_{1}(x)=x, x_{n}(x)=n\left(\left(x 2^{\alpha_{2}(x)} \cdots n^{\alpha_{n}(x)}\right)\right)$.

We will write $\lambda$ for Lebesgue measure on $[0,1)$. The terms independence, random variable, etc., will refer to this probability space.

LEMMA 1. Each $x_{n}$ is uniformly distributed on $[0,1)$.

Proof. This is certainly true for $x$. If it is true for $x_{n}$ then

$$
\begin{aligned}
\lambda\left\{\left[x \mid x_{n+1}(x)<a\right]\right\} \\
=\lambda\left\{\bigcup_{j=0}^{\infty}\left[x \mid x_{n+1}(x) \leqq a, \alpha_{n+1}(x)=j\right]\right\} \\
=\sum_{j=0}^{\infty} \lambda\left\{\left[x \mid x_{n+1}(x) \leqq a, \alpha_{n+1}(x)=j\right]\right\} \\
\quad=\sum_{j=0}^{\infty} \lambda\left\{\left[x \mid n \sum_{k=1}^{j}\left(\frac{1}{n+1}\right)^{k} \leqq x_{n}<n \sum_{k=1}^{j}\left(\frac{1}{n+1}\right)^{k}+\frac{a n}{(n+1)^{j}}\right]\right\} \\
=\sum_{j=0}^{\infty} \frac{a n}{(n+1)^{j}}=a .
\end{aligned}
$$

Received by the editors March 24, 1967.

1 This research was supported in part by the National Science Foundation Grant GP 6216. 
Lemma 2.

$$
\begin{aligned}
& \lambda\left\{\left[x \mid \alpha_{n}(x)=j\right]\right\}=(n-1) / n^{j+1}, \quad j=0,1, \cdots, \\
& \lambda\left\{\left[x \mid \alpha_{n}(x) \geqq j\right]\right\}=1 / n^{j}, \quad j=0,1, \cdots .
\end{aligned}
$$

Proof.

$$
\begin{aligned}
\lambda\left\{\left[x \mid \alpha_{n}(x)=j\right]\right\} & \\
= & \lambda\left\{\left[x \mid(n-1) \sum_{k=1}^{j} \frac{1}{n^{k}} \leqq x_{n-1}<(n-1) \sum_{k=1}^{j+1} \frac{1}{n^{k}}\right]\right\} \\
= & \frac{n-1}{n^{j+1}} .
\end{aligned}
$$

The second formula is an immediate consequence of the first.

Lemma 3. $\alpha_{2}(x)=\alpha_{2}, \alpha_{3}(x)=\alpha_{3}, \cdots, \alpha_{n}(x)=\alpha_{n}$ if and only if

$$
\begin{aligned}
x=\sum_{j=1}^{\alpha_{2}} & \frac{1}{2^{j}}+2^{-\alpha_{2}} \sum_{j=1}^{\alpha_{3}} \frac{1}{3^{j}}+\cdots \\
& +2^{-\alpha_{2}} \cdots(k-1)^{-\alpha_{k-1}} \sum_{j=1}^{\alpha_{k}} \frac{1}{k^{j}}+\cdots \\
& +2^{-\alpha_{2}} \cdots(n-1)^{-\alpha_{n-1}} \sum_{j=1}^{\alpha_{n}} \frac{1}{n^{j}}+2^{-\alpha_{2}} \cdots(n-1)^{-\alpha_{n-1}} \frac{\epsilon}{n-1}
\end{aligned}
$$

where $0 \leqq \epsilon<(n-1) / n^{\alpha_{n}+1}$. In this case $x_{n}(x)=n^{\alpha_{n}} n \epsilon /(n-1)$.

Proof. For $n=2$ it is easily seen that $x=\sum_{j=1}^{\alpha_{2}} 1 / 2^{j}+\epsilon_{2}$ where $0 \leqq \epsilon_{2}<1 / 2^{\alpha_{2}+1}$, and that

$$
x_{2}=2\left(\left(x \cdot 2^{\alpha_{2}}\right)\right)=2^{\alpha_{2}+1} \epsilon_{2} .
$$

Assuming the formulas of the theorem to hold up to $n$ and taking $\alpha_{n+1}$ to be any nonnegative integer we have

$$
\left(\left(x 2^{\alpha_{2}} \cdots n^{\alpha_{n}}\right)\right)=x_{n}(x) / n=n^{\alpha_{n}} \epsilon /(n-1)
$$

so that $\alpha_{n+1}(x)=\alpha_{n+1}$ if and only if

or

$$
\frac{n-1}{n^{\alpha_{n}}} \sum_{j=1}^{\alpha_{n}+1}\left(\frac{1}{n+1}\right)^{j} \leqq \epsilon<\frac{n-1}{n^{\alpha_{n}}} \sum_{j=1}^{\alpha_{n}+1+1}\left(\frac{1}{n+1}\right)^{j}
$$

$$
\epsilon=\frac{n-1}{n^{\alpha_{n}}} \sum_{j=1}^{\alpha_{n}+1}\left(\frac{1}{n+1}\right)^{j}+\epsilon^{\prime}
$$

where 


$$
0 \leqq \epsilon^{\prime}<(n-1) / n^{\alpha_{n}}(1 /(n+1))^{\alpha_{n+1}+1} .
$$

Thus

$$
\begin{aligned}
x=\sum_{j=1}^{\alpha_{2}} \frac{1}{2^{j}} & +\cdots+2^{-\alpha_{2}} \cdots n^{-\alpha_{n}} \sum_{j=1}^{\alpha_{n+1}}\left(\frac{1}{n+1}\right)^{j} \\
& +2^{-\alpha_{2}} \cdots n^{-\alpha_{n}} \frac{\epsilon_{n+1}}{n}
\end{aligned}
$$

where

$$
0 \leqq \epsilon_{n+1}=n^{\alpha_{n}} \frac{n \epsilon^{\prime}}{n-1}<\frac{n}{n+1}\left(\frac{1}{n+1}\right)^{\alpha_{n+1}} .
$$

Also

$$
\begin{aligned}
x_{n+1}(x) & =(n+1)\left(\left(x 2^{\alpha_{2}} \cdots(n+1)^{\alpha_{n+1}}\right)\right) \\
& =(n+1)\left(\left((n+1)^{\alpha_{n+1}} \epsilon_{n+1} / n\right)\right)=(n+1)^{\alpha_{n+1}}(n+1) / n \epsilon_{n+1} .
\end{aligned}
$$

THEOREM 1. The $\alpha_{n}$ are independent and $x_{n}$ is independent of $\alpha_{1}$, $\cdots, \alpha_{n}$. The distributions of these random variables are given in Lemmas 1 and 2.

Proof. Let $\Omega$ be the subset of $[0,1)$ where $x_{n}(x) \leqq a, \alpha_{2}(x)=\alpha_{2}$, $\cdots, \alpha_{n}(x)=\alpha_{n}$ and set

$$
A=\sum_{j=1}^{\alpha_{2}} \frac{1}{2^{j}}+\cdots+2^{-\alpha_{2}} \cdots(n-1)^{-\alpha_{n-1}} \sum_{j=1}^{\alpha_{n}} \frac{1}{n^{j}} .
$$

Then

$$
\begin{aligned}
\lambda\{\Omega\} & =\lambda\left\{\left[x \mid x=A+2^{-\alpha_{2}} \cdots(n-1)^{\alpha_{n-1}} \frac{\epsilon}{n-1}, 0 \leqq \epsilon \leqq a \frac{n-1}{n} n^{-\alpha_{n}}\right]\right\} \\
& =a \frac{1}{n} 2^{-\alpha_{2}} \cdots n^{-\alpha_{n}}=a \prod_{j=2}^{n}\left(\frac{j-1}{j^{\alpha j+1}}\right) \\
& =\lambda\left\{\left[x \mid x_{n}(x) \leqq a\right]\right\} \prod_{j=2}^{n} \lambda\left\{\left[x \mid \alpha_{j}(x)=\alpha_{j}\right]\right\} .
\end{aligned}
$$

THEOREM 2. For almost every $x$,

(1) $\alpha_{n}(x)$ takes only the values 0 and 1 from some point on,

(2) the value 1 occurs infinitely often,

(3) for infinitely many $n, \alpha_{k}(x)=0$ for $A_{n} \leqq k<A_{n+1}, A_{n}$ being any increasing sequence with $\sum_{n=2}^{\infty} A_{n} / A_{n+1}=\infty$. 
Proof.

$$
\sum_{n=2}^{\infty} \lambda\left\{\left[x \mid \alpha_{n}(x) \geqq 2\right]\right\}=\sum_{n=2}^{\infty} \frac{1}{n^{2}}<\infty
$$

so according to the Borel-Cantelli theorem the event $\alpha_{n}(x) \geqq 2$ can occur only finitely often for almost every $x$. If only finitely many 1 's occur then $\alpha_{n}(x)=0$ from some point on and $x$ must be rational. If we set

$$
\Omega_{n}=\left[x \mid \alpha_{k}(x)=0, A_{n} \leqq k<A_{n+1}\right]
$$

then the sets $\Omega_{n}$ are independent and

$$
\lambda\left\{\Omega_{n}\right\}=\prod_{k=A_{n}}^{A_{n}+1-1} \frac{k-1}{k}=\frac{A_{n}-1}{A_{n+1}-1} .
$$

Thus $\sum_{n=0}^{\infty} \lambda\left\{\Omega_{n}\right\}=\infty$, so by the Borel-Cantelli theorem almost every $x$ is in infinitely many $\Omega_{n}$.

If we set $Q_{n}(x)=2^{\alpha_{2}} \cdots n^{\alpha_{n}}$ then, for some integer $P_{n}(x)$,

$$
x=P_{n}(x) / Q_{n}(x)+(1 / n) x_{n} / Q_{n}(x) .
$$

Our final theorem concerns the asymptotic distribution of $x_{n}$ and $Q_{n}$.

THEOREM 3. For every $n$

$$
x=P_{n}(x) / Q_{n}(x)+(1 / n) x_{n}(x) / Q_{n}(x)
$$

where

(i) $P_{n}(x)$ is an integer,

(ii) $Q_{n}(x)$ is an integer whose prime factors are all $\leqq n$,

(iii) $x_{n}(x)$ is uniformly distributed on $[0,1)$ and is independent of $Q_{n}(x)$,

$$
Q_{n}(x)=\left(\prod_{j=2}^{n} k^{1 /(k-1)}\right)
$$

$$
\cdot \exp \left(\theta_{n}(x)\left[\sum_{j=2}^{n}\left(\log ^{2} k\right)\left(\frac{1}{k-1}+\frac{1}{(k-1)^{2}}\right)\right]^{1 / 2}\right)
$$

where, as $n \rightarrow \infty$,

$$
\lambda\left\{\left[x \mid \theta_{n}(x) \leqq a\right]\right\} \rightarrow \frac{1}{(2 \pi)^{1 / 2}} \int_{-\infty}^{a} e^{-t^{2} / 2} d t .
$$

Proof. Only (iv) needs to be proved. Straightforward calculations show 


$$
\begin{gathered}
\int_{0}^{1} \alpha_{k}(x) \log k d x=\frac{\log k}{k-1}, \\
\int_{0}^{1}\left(\alpha_{k}(x)-\frac{1}{k-1}\right)^{2} \log ^{2} k d x=\left(\log ^{2} k\right)\left(\frac{1}{k-1}+\left(\frac{1}{k-1}\right)^{2}\right), \\
\int_{0}^{1}\left|\alpha_{k}(x)-\frac{1}{k-1}\right|^{3} \log ^{3} k d x \leqq C \frac{\log ^{3} k}{k-1} .
\end{gathered}
$$

Since

$$
\tau_{n}^{3}=\sum_{k=2}^{n} \int_{0}^{1}\left|\alpha_{k}(x)-\frac{1}{k-1}\right|^{3} \log ^{3} k d x \leqq C_{1} \log ^{4} n
$$

and

$$
\sigma_{n}^{2}=\sum_{k=2}^{n} \int_{0}^{1}\left|\left(\alpha_{k}(x)-\frac{1}{k-1}\right)\right|^{2} \log ^{2} k d x \geqq C_{2} \log ^{3} n,
$$

$\tau_{n} / \sigma_{n} \rightarrow 0$ as $n \rightarrow \infty$ so we can apply Liapounov's theorem with $\delta=1$ $[1$, Theorem 4.4 , p. 144$]$ to conclude that

$$
\theta_{n}=\frac{1}{\sigma_{n}} \sum_{k=2}^{n}\left(\alpha_{k}(x)-\frac{1}{k-1}\right) \log k
$$

has the asymptotic distribution required in (iv). This gives

$$
\log Q_{n}=\sum_{k=2}^{n} \frac{\log k}{k-1}+\sigma_{n} \theta_{n}
$$

and

$$
Q_{n}=\left(\prod_{k=2}^{n} k^{1 /(k-1)}\right) \exp \left(\sigma_{n} \theta_{n}\right)
$$

\section{BIBLIOGRAPHY}

1. J. L. Doob, Stochastic processes, Wiley, New York, 1953.

2. E. Harzheim, An elementary number theoretical remark, Proc. Amer. Math. Soc. 17 (1966) 534-535.

University of Southern California 